\title{
Intelligent environment for monitoring Alzheimer patients, agent technology for health care
}

\author{
Juan M. Corchado, Javier Bajo *, Yanira de Paz, Dante I. Tapia \\ Departamento Informática y Automática Universidad de Salamanca, Plaza de la Mercaed s/n, 37008, Salamanca, Spain
}

Received 18 August 2006; received in revised form 9 March 2007; accepted 30 April 2007

\section{Abstract}

This paper presents an autonomous intelligent agent developed for monitoring Alzheimer patients' health care in execution time in geriatric residences. The AGALZ (Autonomous aGent for monitoring ALZheimer patients) is an autonomous deliberative casebased planner agent designed to plan the nurses' working time dynamically, to maintain the standard working reports about the nurses' activities, and to guarantee that the patients assigned to the nurses are given the right care. The agent operates in wireless devices and is integrated with complementary agents into a multi-agent system, named ALZ-MAS (ALZheimer Multi-Agent System), capable of interacting with the environment. AGALZ description, its relationship with the complementary agents, and preliminary results of the multi-agent system prototype in a real environment are presented.

(C) 2007 Published by Elsevier B.V.

Keywords: Multi-agent system; Deliberative agent; Case-based reasoning; RFID; Health care

\section{Introduction}

Agents and multi-agent systems (MAS) have become increasingly relevant for developing distributed and dynamic open systems. The AGALZ (Autonomous aGent for monitoring ALZheimer patients) agent is aimed to improve the efficiency of health care in geriatric residences. This paper describes the AGALZ agent and explains how this deliberative planning agent has been designed and implemented. The AGALZ agent has been integrated within a multi-agent system called ALZ-MAS (ALZheimer Multi-Agent System), devel-

\footnotetext{
* Corresponding author.

E-mail addresses: corchado@usal.es (J.M. Corchado), jbajope@usal.es (J. Bajo), yanira@usal.es (Y. de Paz), dantetapia@usal.es (D.I. Tapia).
}

oped for facilitating the management and control of geriatric residences. The aim of this paper is to present the AGALZ agent and to demonstrate how its planning mechanism improves the medical assistance in geriatric residences by optimizing the visiting schedules. These agents also facilitate the nurses' and doctors' work by providing updated information about patients and emergencies, as well as historical data.

The applications of agents and multi-agent systems in the health care and clinical management environments are becoming a reality; this fact has been reported for example by Foster et al. [7]. Most agent-based applications are related to the use of this technology in patient monitoring, treatment supervision and data mining. Lanzola et al. [11] present a methodology that facilitates the development of interoperable intelligent software agents for medical applications and propose a 
generic computational model for implementing them. The model may be specialized in order to support all the different information and knowledge related requirements of a hospital information system. However, they do not contemplate the possibility of using wireless and RFID technology as in this paper, nor have they proposed the use of dynamic planning. Others, such as Jeffrey and Meunier [13] propose the use of virtual machines supporting mobile software agents using the functional programming paradigm. This virtual machine provides the application developer with a rich and robust platform upon which to develop distributed mobile agent applications, specifically when targeting distributed medical information and distributed image processing. This interesting proposal is not viable due to the security reasons that affect mobile agents, and they have not defined an alternative for locating patients or generating planning strategies. There are also agentbased systems that help patients to get the best possible treatment and remind the patient about follow-up tests [14]. They assist the patient in managing continuing ambulatory conditions (chronic problems). They also provide health-related information by allowing the patient to interact with the on-line health care information network. Others such as Decker and Li [6], propose a system to increase hospital efficiency using global planning and scheduling techniques. They propose a multi-agent solution using the generalized partial global planning approach that preserves the existing human organization and authority structures, while providing better system-level performance (increased hospital unit throughput and decreased patient stay time). To do this, they extend the proposed planning method with a coordination mechanism to handle mutually exclusive resource relationships, using resource constraint scheduling. This system does not use dynamic planning, it uses a static task assignment, and it does not work on wireless devices and does not use location information or RFID technology.

The AGALZ agents are integrated within ALZ-MAS multi-agent system which is a dynamic system for the management of different aspects of the geriatric center. This distributed system uses Radio Frequency Identification (RFID) [16] technology for ascertaining patients' location in order to maximize their safety or to generate medical staff plans. The development of such multiagent system has been motivated for one of the more distinctive characteristics of geriatric or Alzheimer residences, which is their dynamism, in the sense that the patients change very frequently (new patients arrive and others pass away), while the staff rotation is also relatively high and they normally work in shifts of eight hours. ALZ-MAS provides the personnel of the residence with updated information about the center and the patients, provides the working plan, information about alarms or potential problems and keeps track of their movements and actions within the center. Dynamic problems require the dynamic solutions provided by this technology. From the user's point of view the complexity of the solution has been reduced with the help of friendly user interfaces and a robust and easy to use multi-agent system.

The proposed planning agent AGALZ is a deliberative one and uses a case-based reasoning (CBR) [1] architecture, that allows it to respond to events, to take the initiative according to its goals, to communicate with other agents, to interact with users, and to make use of past experiences to find the best plans to achieve goals. This particular agent uses a special type of CBR systems which we call Case-Based Planning (CBP) system, specially designed for planning construction. AGALZ is also a deliberative agent that works at a high level with the concepts of Believe, Desire, Intention (BDI) [3]. The AGALZ can be called a CBP-BDI agent, and has learning and adaptation capabilities, which facilitates its work in dynamic environment. A CBP-BDI agent is therefore a particular type of CBR-BDI agent [5], which uses case-based reasoning as a reasoning mechanism, which allows it to learn from initial knowledge, to interact autonomously with the environment as well as with users and other agents within the system, and to have a large capacity for adaptation to the needs of its surroundings.

There is an ever growing need to supply constant care and support to the disabled and elderly [15] and the drive to find more effective ways to provide such care has become a major challenge for the scientific community. During the last three decades the number of Europeans over 60 years old has risen by about $50 \%$. Today they represent more than $25 \%$ of the population and it is estimated that in 20 years this percentage will rise to one third of the population, meaning 100 millions of citizens [4]. This situation is not exclusive to Europe, since studies in other parts of the world show similar tendencies. In the United States of America, people over 65 years old are the fastest growing segment of the population and it is expected that in 2020 they will represent about 1 of 6 citizens totaling 69 million by 2030 [9]. Furthermore, over $20 \%$ of people over 85 years old have a limited capacity for independent living, requiring continuous monitoring and daily care. The Institute of Medicine has studied the role of information technology in improving health care delivery in the US. In [12], the Institute presents a strategy and an action plan to foster innovation and 
151 improve the delivery of care. The need to reinvest in the

152 system is underlined and as such six health care aims are 153 defined (to be safe, effective, patient-centered, timely, 154 efficient and equitable) and ten guidelines for the 155 redesign of the system are given focusing on the role 156 of the patient and improvements in knowledge, 157 communication and safety mechanisms. Moreover the 158 Institute proposes a strategy to improve safety in health 159 care based on the study of medical errors [9]. The 160 proposed system presented here has been conceived and 161 developed taking these considerations into account.

162 The importance of developing new and more reliable 163 ways to provide care and support to the elderly is 164 underlined by this trend [4], and the creation of secure, 165 unobtrusive and adaptable environments for monitoring 166 and optimizing health care will become vital. Some 167 authors [15] consider that tomorrow's health care 168 institutions will be equipped with intelligent systems 169 capable of interacting with humans. Multi-agent systems 170 and architectures based on intelligent devices have 171 recently been explored as supervision systems for medical 172 care for the elderly or Alzheimer patients [7], these 173 intelligent systems aim to support them in all aspects of 174 daily life, predicting potential hazardous situations and 175 delivering physical and cognitive support. Multi-agent systems together with the use of RFID technology offer new possibilities and open new fields such as the ambient intelligence that may facilitate the integration of distributed intelligence software applications in our daily life.

Radio Frequency Identification (RFID) [16] is an automated data-capture technology that can be used to electronically identify, track, and store information about products, items, components or people. It is most frequently used in industrial/manufacturing, transportation, distribution, and warehousing industries, but there are other growth sectors including health care [16]. ALZ-MAS uses microchips mounted on bracelets worn on the patient's wrist or ankle, and sensors installed over protected zones, with an adjustable capture range up to $2 \mathrm{~m}$. The microchips or transponders use a $125 \mathrm{kHz}$ signal help locate the patients, which can be ascertained by consulting the AGALZ agents installed in personnel PDAs.

The following section presents the AGALZ agent and its planning strategy. Section 3 shows the Multiagent system ALZ-MAS in which AGALZ has been incorporated. Section 4 presents the results obtained with the system and the conclusion.

\section{AGALZ: autonomous health care agent}

AGALZ is an autonomous deliberative case-based planner (CBP-BDI) agent developed for integration within a multi-agent system named ALZ-MAS. The goal of this agent is to provide efficient working schedules, in execution time, for geriatric residences staff and therefore to improve the quality of health care and the supervision of patients in geriatric residences. Each of the AGALZ agents is assigned to a nurse or a doctor of a residence, and provides also information about patient locations, historical data and alarms. As the members of the staff are carrying out their duties (following the plan provided by the agent) the initial proposed plan may need to be modified due for example to delays or alarms, in this case the agent is capable of replanning in execution time. The internal structure and capabilities of the AGALZ agents are based on the mental aptitudes of beliefs, desires, and intentions. This high level structure facilitates the incorporation of CBR systems [1] as a deliberative mechanism within BDI agents, facilitating learning and adaptation and providing a greater degree of autonomy than pure BDI architecture [5].

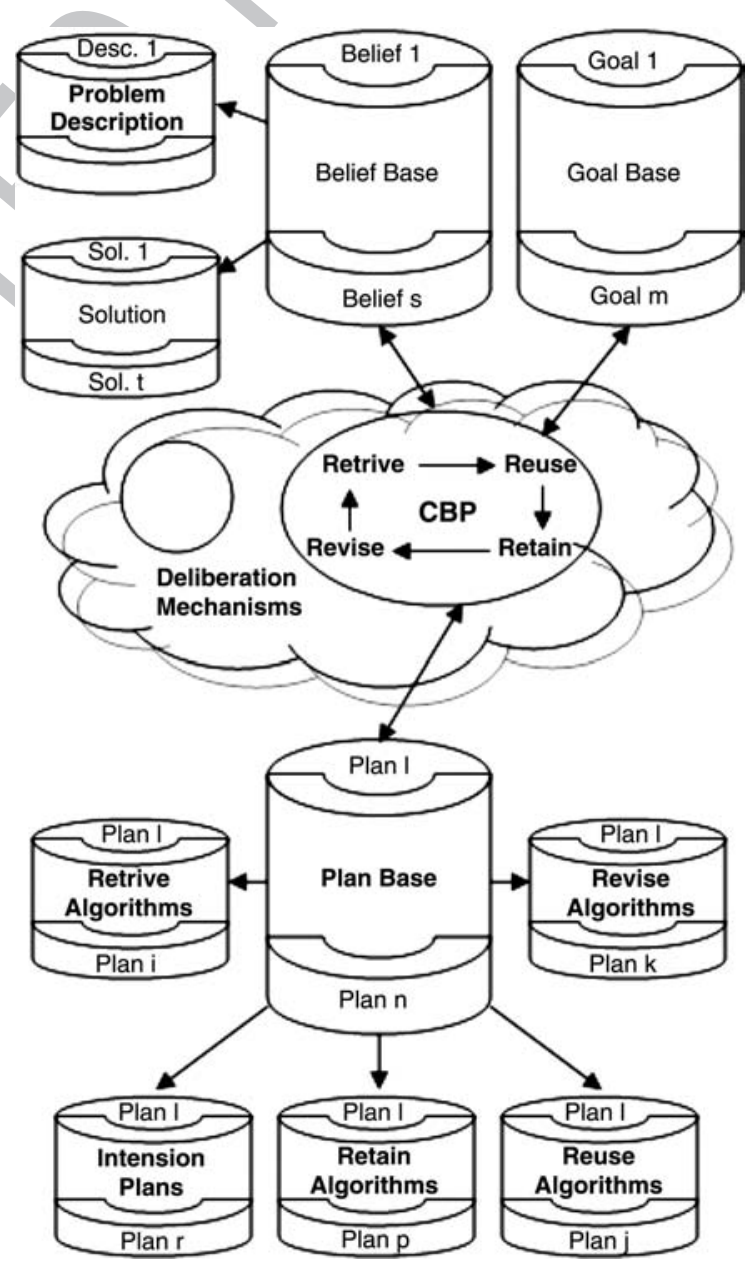

Fig. 1. AGALZ internal structure. 
To introduce a CBR motor into a BDI agent it is 221 necessary to represent the cases used in a CBR system 222 by means of beliefs, desires and intentions, and 223 implement a CBR cycle. A case is a past experience 224 composed of three elements: an initial state or problem 225 description that is represented as a belief; a final state 226 that is represented as a set of goals; and the sequence of 227 actions that makes it possible to evolve from an initial 228 state to a final state. This sequence of actions is 229 represented as intentions or plans. In a planning agent, 230 the reasoning motor generates plans using past experi231 ences and planning strategies, so the concept of Case232 Based Planning is obtained [8]. CBP consists of four 233 sequential stages: retrieve stage to recover the most 234 similar past experiences to the current one; reuse stage to 235 combine the retrieved solutions in order to obtain a 236 new optimal solution; revise stage to evaluate the ob237 tained solution; and retain stage to learn from the new 238 experience.

239 Fig. 1 shows the internal structure of a CPB-BDI 240 agent. Problem description (initial state) and solution 241 (situation when final state is achieved) are represented 242 as beliefs, the final state as a goal (or set of goals), and 243 the sequences of actions as plans. The CBP cycle is 244 implemented through goals and plans. When the goal 245 corresponding to one of the CBP stages is triggered, 246 different plans (algorithms) can be executed concur247 rently to achieve the goal. Each plan can trigger new 248 sub-goals and, consequently, cause the execution of 249 new plans. AGALZ is an autonomous agent that can 250 survive in dynamic environment because incorporates 251 this planning mechanism. The following subsection 252 presents the planning structure of the AGALZ agent.

\section{3. The planning model of the AGALZ agent}

254 A deliberative CBP-BDI agent is specialized in 255 generating plans in execution time and incorporates a 256 case-based planning (CBP) reasoning mechanism. The 257 purpose of the CBR agent is to solve new problems by 258 adapting solutions that have been used to solve similar 259 problems in the past [1], and the CBP-BDI agents are 260 a variation of the CBR-BDI agents, based on the plans 261 generated from each case. The AGALZ agents require 262 dynamic planning systems that allow them to respond 263 to changes in the environment and to provide efficient 264 plans in execution time for optimizing the working 265 rotas. The CBP planner used by the AGALZ agent 266 identifies a plan, for a given nurse, to provide daily 267 nursing care in the residence. It is very important to 268 maintain a map with the location of the different 269 patients at the time of planning or replanning, which is why RFID technology is used to facilitate the location and identification of patients, nurses and doctors.

So as not to overload the mathematical formula without losing generality, this section explains how the most replannable plan is chosen, focussing on one nurse and one patient. Let $E=\left\{e_{0}, \ldots, e_{\mathrm{n}}\right\}$ the set of tasks that the nurse is assigned to.

$a_{j}: \underset{e_{i}}{E} \rightarrow \underset{a_{j}\left(e_{i}\right)=e_{j}}{E}$

An agent plan is the name given to a sequence of actions (1) that, from a current state $e_{0}$, defines the path of states through which the agent passes in order to offer the nurses the optimum path according to each of their characteristics. Below, in Eq. (2), the dynamic relationship between the behaviour of the agent and the changes in medium is modelled. The behaviour of agent $A$ can be represented by its action function $a_{A}(t) \forall t$, defined as a correspondence between one moment in time $t$ and the action selected by the agent,

Agent $A=\left\{a_{\mathrm{A}}(t)\right\}_{t \in b T \subseteq N}$

From the definition of the action function $a_{A}(t)$ it is possible to define a new relationship that collects the idea of an agent's action plan (3),

$P_{A}: \underset{\left(t, a_{A}(t)\right) \rightarrow P_{A}(t)}{\operatorname{Tx} A}$

in the following way,

$P_{A}\left(t_{n}\right)=\sum_{i=1}^{n} a_{i A}\left(t_{i}-t_{i-1}\right)$

Given the dynamic character desired for the planning agent, for a definition of the agent plan, the continuous extension of the previous expression (4) is proposed, in other words (Eq. (5))

$P_{A}\left(t_{n}\right)=\int_{t_{0}}^{t_{n}} a_{A}(t) \mathrm{d} t$

The variation of the agent plan $p_{A}(t)$ will be provoked essentially by: The changes that occur in the environment and that force the initial plan to be modified, and the knowledge from the success and failure of the plans that were used in the past, and which are favoured or punished via learning. $O$ indicates the objectives of the agent and $O^{\prime}$ are the results achieved by the plan. $R$ are the total resources and $R$ ' are the resources consumed by the agent. The
296

270

271

272

273

274

275

276

279

280

281

282

283

284

285

286

287

288

289

290

292

293

294

295

299

300

301

302

305

306

307

308

309

310

311

312

313 
314 efficiency of the plan (6) is the relationship between 315 the objectives attained and the resources consumed

$316 E_{\mathrm{ff}}=\frac{\#\left(O^{\prime} \cap O\right)}{\# R^{\prime}}$.

318 Where \# means cardinal of a set. The objective is to 319 introduce an architecture for a planning agent that 320 behaves - and selects its actions - by considering the 321 possibility that the changes in the environment block 322 the plans in progress. This CBP-BDI agent searches 323 continually for the plan that can most easily be 324 replanned in the event of interruption (the most re325 plan-able intention). Given an initial point $e_{0}$, the term 326 planning problem is used to describe the search for a 327 way of reaching a final point $e_{i} \equiv e^{*} \in E$ that meets a 328 series of requirements. Given a discrete variable $X$ that 329 can take values of a numerable set represented as

$330 X=\left\{x_{i}\right\}_{i \in N}$.

It is possible to define the associated accumulated variable (8), that can be denoted as $\operatorname{Ac}(X)$, for a new variable that is constructed by assigning each of the possible values $x_{i}$ taken by variable $X$, the total of previous results. If $X$ is discrete, the value $i$-th of the variable $\operatorname{Ac}(X)$ is defined as

$\operatorname{Ac}\left(x_{i}\right)=\sum_{j=1}^{i} x_{j} \quad \forall x_{i} \in X$.

If the variable $X$ is continuous with values in the 341 interval $[a, b]$, it is represented by function $x(t)$; the 342 variable $\operatorname{Ac}(X)$ at a point $x_{i} \in[a, b]$ is defined:

$\operatorname{Ac}\left(x_{i}\right)=\int_{a}^{x_{i}} x(t) \mathrm{d} t \quad \forall x_{i} \in[a, b]$.

Given a problem $E$ and a plan $p(t)$ the functions $\mathrm{Ob}$ and Rc accumulated from the objectives and costs of the plan (10) can be constructed. For all time points $t_{i}$ two variables can be associated:

$\mathrm{Ob}\left(t_{i}\right)=\int_{a}^{t_{a}} O(t) \mathrm{d} t \operatorname{Rc}\left(t_{i}\right)=\int_{a}^{t_{i}} R(t) \mathrm{d} t$.
This allows us to construct a planning space (or space representing the environment for planning problems) as a vectorial hyper dimensional space where each axis represents the accumulative variable associated with each objective and resource. The planning space, defined in this way, conforms to the following properties:

1. The representations of the plans within the planning space are always monotonously growing functions. Given that $\mathrm{Ob}(t)$ and $\operatorname{Rc}(t)$ are functions defined as positive, function $p(t)$ expressed at these coordinates is constant or growing.

2. In the planning space, the straight lines represent plans of constant efficiency. If the representations of the plans are straight lines, the slope of the function is constant, and coincides with the definition of the efficiency of the plan.

$\frac{\mathrm{d}}{\mathrm{d} t} p t=$ cte $\Longleftrightarrow \lim _{\Delta \rightarrow 0} \frac{\Delta O(t)}{\Delta R(t)}=$ cte.

In an $n$-dimensional space, the extension of the straight concept line is called a geodesic curve. In this sense the notion of geodesic plans can be introduced, defined as those that maintain efficiency at a constant throughout their development.

The concept of a geodesic plan can be better understood through the idea of a "plan of minimum risk". Given a problem, the agent must search for the plan that determines a solution with a series of restrictions $F(O ; R)=0$. In the plans base those plans that are initially compatible with the problem faced by the agent, with the requirements imposed on the solution according to the desires, and in the current state [1] are sought. If all the possible plans $\left\{p_{1}, \ldots, p_{n}\right\}$ are represented within the planning space, a subset of states that the agent has already attained in the past will be obtained in order to resolve similar problems.

With the mesh of points obtained (generally irregular) within the planning space and using interpolation techniques, a working hyper plan $h(x)$ (that encapsulates the information on the set of restrictions from restored experiences, giving place by definition to an hyper plan due to verifies $h\left(x_{j}\right)=p_{j} j=1, \ldots, n$ and the planning space is the dimension $n$ ) can be obtained, from which geodesic plans can be calculated and with which variation calculation is applied. Suppose, for simplicity's sake, a planning space of dimension 3 with coordinates $\left\{O, R_{1}\right.$, $\left.R_{2}\right\}$. Between the point $e_{0}$ and the objective points $f_{\mathrm{s}} f=\left\{e_{1}\right.$, $\left.\ldots, e_{m}\right\}$ and over the interpolation surface $h(x)$, the Euler Theorem [8] guarantees that the expression of the geodesic plans will be obtained by resolving the system of equations in Eq. (12):

$$
\left\{\begin{array}{l}
\frac{\partial L}{\partial R_{1}}-\frac{\mathrm{d}}{\mathrm{d} O} \frac{\partial L}{\partial R_{1}^{\prime}}=0 \\
\frac{\partial L}{\partial R_{2}}-\frac{\mathrm{d}}{\mathrm{d} O} \frac{\partial L}{\partial R_{2}^{\prime}}=0
\end{array} .\right.
$$

370 371 372 373 374 375 376 377 378 379 380 381 382 383 384 385 386 387 388 389 390 391 392 393 394 395 396 397 398 399 400 
Where $R_{i}$ is the function accumulated $R, O$ is the function of accumulated $O$ and $L$ is the distance function on the hyper plan $h(x)$,

$L=\int_{h} \mathrm{~d} l$.

In order to obtain all the geodesic plans that, on the surface $h(x)$ and beginning at $e_{0}$, allow us to reach any of the points $e^{*} \in f_{\mathrm{s}} f$, a condition of the surrounding must be imposed: the initial point will be $e_{0}=\left(O_{0}, R_{0}\right)$.

Using variation techniques expressions for all the geodesic plans that, beginning at $e_{0}$ allow us to attain the desired point are obtained. Once plans have been obtained that will create efficient solutions between the current state and the set of solution states, we will be able to calculate the plan around it (along its trajectory) by a denser distribution of geodesic plans (a greater number of geodesic plans in its environment). The tool that allows us to determine this is called the minimum Jacobi field associated with the solution set. $g_{0}:[0,1] \rightarrow S$ be a geodesic over a surface $S$. Let $h:[0,1] x[-\varepsilon, \varepsilon] \rightarrow S$ be a variation of $g_{0}$ so that for each $t \in(-\varepsilon, \varepsilon)$, the set $\left\{h_{t}(s)\right\}_{t \in(-\varepsilon, \varepsilon)}: h_{t}(s)$ for all $t \in(-\varepsilon, \varepsilon)$ are geodesic in $S$ and they begin at $g_{0}(0)$, in other words, they conform to $h_{t}(0)=g_{0}(0)$ for all $t \in(-\varepsilon, \varepsilon)$. In these conditions, taking the variations to a differential limit, the Eq. (14) is obtained:

$$
\begin{aligned}
\lim _{t \rightarrow 0}\left\{h_{t}(s)\right. & \left.=g_{0}(s+t)=\lim _{t \rightarrow 0} h(s, t)\right\}=\left.\frac{\partial g_{0}}{\partial t}\right|_{s, 0} \\
& =\frac{\mathrm{d} g_{0}}{\mathrm{~d} s} \equiv J_{g 0}(s) .
\end{aligned}
$$

The term $J_{g 0}(s)$ is given to the Jacobi Field of the geodesic $g_{0}$ for the set $\left\{g_{n}(x)\right\}_{n \in N}$, and in the same way that the definition has been constructed, it is possible to give a measurement for the distribution of the other geodesics of $\left\{g_{n}(x)\right\}_{n \in N}$ around $g_{0}$ throughout the trajectory. Given a set of geodesics, some of them are always $g^{*}$ that, in their environment, have a greater distribution than other geodesics in a neighbouring environment. This is equivalent to say that it presents a variation in the distribution of geodesics lower than the others and therefore the Jacobi Field associated with $\left\{g_{n}(x)\right\}_{n \in N}$ reaches its lowest value at $J_{g^{*}}$.

Let's return to the problem of identifying the most replan-able intention, following the recuperation and variation calculation phase, contains a set of geodesic plans $\left\{p_{1}, \ldots, p_{n}\right\}$. If the $p^{*}$ is selected with a minimum Jacobi Field value, it can be guaranteed that in the event of interruption it will have around it a greater number of geodesic plans in order to continue. This suggests that given a problem with certain restrictions $F(O ; R)=0$, the geodesic plan $p *$ with minimum associated Jacobi field associated with the set $\left\{g_{n}(x)\right\}_{n \in N}$ is called the most replan-able solution. The behaviour model $G$ for the CBPBDI agent is Eq. (15).

$$
G\left(e_{0}, p_{1}, \cdots, p_{n}\right)=p^{*} \Longleftrightarrow \exists_{n \in N} N / J_{g_{n}} \equiv J_{g^{*}}=\operatorname{Min}_{n \in N} J_{g_{n}} .
$$

If the plan $p *$ is not interrupted, the agent will reach a desired state $e_{j} \equiv e^{*} \in f_{\mathrm{s}} f, j \in\{1, \ldots, m\}$. In the learning phase, a weighting $w_{f}(p)$ is stored. With the updating of weighting $w_{f}\left(p^{*}\right)$, the planning cycle of the CBP motor is completed.

Let's suppose that the agent has initiated a plan $p^{*}$ but at a moment $t>t_{0}$, the plan is interrupted due to a change in the environment. The geodesic planning meets the conditions of the Bellman Principle of Optimality [2], in other words, each on of the plan's parts is partially geodesic between the selected points. This guarantees that if $g_{0}$ is geodesic for interrupted $e_{0}$ in $t_{1}$, because $e_{0}$ changes to $e_{1}$, and $g_{1}$ is geodesic to $e_{1}$ that is begun in the state where $g_{0}$ has been interrupted, it follows that: $g=g_{0}+g_{1}$ is geodesic to $e=e_{0}\left(t_{1}-t_{0}\right)+e_{1}\left(t_{2}-t_{1}\right)$, the dynamic process follows the CBP cycle recurrently: each time a plan finds itself interrupted; it generates from the state reached so far, the surroundings of the plans from the case base and adjusts them to the new problem. With this it calculates the geodesic plans and selects the one which meets the minimum conditions of the associated Jacobi field.

A minimum global Jacobi field $J(t)$ also meets Bellman's conditions of optimality [2], in other words, a minimum global Jacobi field, must select minimum Jacobi fields "in pieces".

$J_{\min }(t)=\left\{J_{\min }\left(t_{1}-t_{0}\right), J_{\min }\left(t_{2}-t_{1}\right), \cdots, J_{\min }\left(t_{n}-t_{n-1}\right)\right\}$.

If on the one hand, successive Jacobi fields generate one Jacobi field, and on the other hand, minimum Jacobi fields generate a minimum Jacobi field, the MRPI agent that follows a strategy of replanning $G(t)$ as indicated to survive a dynamic environment, it generates a global plan $p^{*}(t)$ that, faced with all possible global plans $\left\{p_{n}(t)\right\}_{n \in N}$, presents a minimum value in its Jacobi field $J_{g} *(t) \equiv J_{p} *(t)$. The AGALZ agent is a CBR-BDI agent that seeks plans in a dynamic environment in execution time.

4. ALZ-MAS: a multi-agent environment for the AGALZ agent

The Alzheimer Santísima Trinidad Residence of Salamanca has been interested in improving the services 
offered to its patients and has collaborated in the development of the technology presented here, providing their know-how and experimenting with the prototype developed. This residence is intended for people over 65 years old, and has the following services and facilities among others: TV room, geriatric bathroom, hairdressing salon, medical service, religious attention, occupational therapy, technical assistance, terrace, garden, laundry service, clothes adjustment, infirmary, reading room, living room, room of visits, cafeteria, social worker, chapel, elevator, customized diet, and multipurpose room.

Fig. 2 shows a diagram of the first floor of the Santísima Trinidad Residence of Salamanca containing the main facility rooms, while all the patients' rooms are located in the second floor. This residence has capacity for 60 patients, an average of 6 nurses, one social worker and 5 more employees with other responsibilities. We selected 30 patients to test the system, so the hardware implemented at the Residence basically consisted of 42 ID door readers (Hitag HT RM401and mobile WorkAbout Pro RFID), one on each door and elevator, 4 controllers, one at each exit, one in the first floor hall and another in the second floor hall, and 36 bracelets (Sokymat ID Band Unique Q5 with a chip Hitag S 256), one for each patient and the nurses. The ID door readers

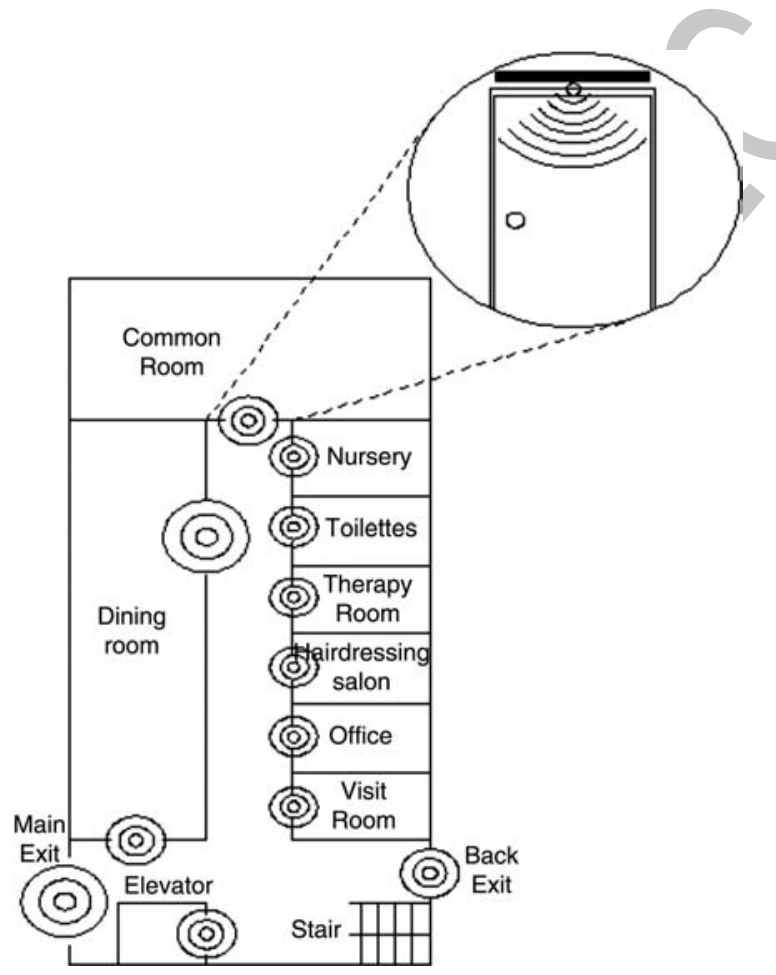

Fig. 2. Sensor positioning in the first floor of the Santísima Trinidad Residence of Salamanca. get the ID number from the bracelets and send the data to the controllers which send a notification to the Manager agent.

The ALZ-MAS multi-agent system is a distributed system of a relatively high dimension, and requires a detailed analysis and design process before its design. We decided to use a combination of Gaia [18] and AUML for the system design, in an attempt to take advantage of both. Through the Gaia analysis, two models are obtained: the role model and the interaction model. Studying the requirements of the problem, five roles have been chosen: the Patient role manages the patient's personal data and behaviour (monitoring, location, daily tasks, and anomalies); the Doctor role treats patients; the Nurse role schedules the nurse's working day obtaining dynamic plans depending on the tasks needed for each assigned patient; the Security role controls the patients' location and manages locks and alarms; and finally, the Manager role manages the medical record database and the doctor-patient and nurse-patient assignment.

As far as an interaction model is concerned, the dependences and relations between roles are described. For the roles involved in the system a number protocols have been considered: request a treatment, inform about monitoring data, inform about care results, request a doctor assignment, request a nurse assignment, inform about assignment, request a patient's daily plan, inform about a patient's daily tasks, request a patient location, inform a nurse about a lock activation, report alarm activation, request doctor situation, doctor reports on his schedule, request a nurse situation, nurse reports situation, patient reports an anomaly, patient reports on personal data and previous medical records. For example, when the nurse wants to know the tasks required for the patient, the Nurse role executes a protocol RequestPatientPlanif through which is able to make a request to the Patient role. The Patient role acts to give a suitable response to the Nurse role and executes the InformPlanif protocol to communicate the planned tasks to the NURSE role.

In the Gaia design process three models are considered: agent model, services model and acquaintance model [18].

To achieve a low level AUML design, with enough details for an implementation to be carried out. The AUML design provides class diagrams for each agent, collaboration or sequence diagrams for each interaction, state and activity diagrams to represent internal states and protocol diagrams to model communicative acts. Some examples of the low level AUML detailed design will be presented in the next section. 
The conclusions obtained after the analysis and design process let us conclude that ALZ-MAS is composed of four different types of agent:

- Patient agent manages the patient's personal data and behaviour (monitoring, location, daily tasks, and anomalies). Every hour validates the patient location, monitors the patient state and sends a copy of its memory base (patient state, goals and plans) to the manager agent in order to maintain backups. The patient state is instantiated at execution time as a set of beliefs and these beliefs are controlled through goals that must be achieved or maintained. The beliefs that were seen to define a general patient state at the Santísima Trinidad Residence of Salamanca were: weight, temperature, blood pressure, feeding (diet characteristics and next time to eat), oral medication, parenteral medication, posture change, toileting, personal hygiene, and exercise. The beliefs and goals used for every patient depend on the plan (treatment) or plans that the doctors prescribe. The patient agent monitors the patient state by means of the goals. To know if a goal has been achieved or has failed, it is necessary to maintain continuous communication with the rest of the ALZ MAS agents. At least once per day, depending on the corresponding treatment, the patient agent must contact the nurse agent. The patient agent must have periodic communication with the doctor agent. Finally the patient agent must ensure that all the actions indicated in the treatment are taken out.

- Manager agent plays two roles the Security role that controls the patients' location and manages locks and alarms; and the Manager role that manages the medical record database and the doctor-patient and nurse-patient assignment. It must provide security for the patients and medical staff and the patients, doctors and nurse assignment must be efficient.

- Doctor AGALZ agent treats patients. The Doctor agent needs to interact with the Patient agent to order a treatment and receive periodic reports, with the Manager agent to consult medical records and assigned patients, and with AGALZ agent to ascertain the patient evolution.

- AGALZ agent schedules also the nurse's working day obtaining dynamic plans depending on the tasks needed for each assigned patient. AGALZ manages nurses' profiles, tasks, available time and resources. The generated plans must guarantee that all the patients assigned to the nurse are given care. The nurse cannot exceed 8 working hours. Every agent generates personalized plans depending on the nurse's profile and working habits.
Manager and Patient agents run in a central computer, but AGALZ agents run on mobile devices, so a robust wireless network has been installed as an extension to the existing wired LAN. With respect to the question of failure recovery, a continuous monitoring of the system is carried out. Every agent saves its memory (personal data) onto a data base. The most sensitive agents are patient agents, so these agents save their state every hour. When an agent fails, another instance can be easily created from the latest backup. The database and server used must have redundancy and failure recovery, so a RAID (Redundant Array of Inexpensive Disks) server is used. In the case of a server failure, an alarm is generated and all the plans and information required for nurses and doctors to carry out their working day are automatically printed. A secure and authenticated access to the patients data is provided. The use of different authorisations for users, logins and passwords, and the encryption of messages using a public key infrastructure and SSL (Secure Socket Layer) have already been implemented. Moreover, the RFID tag only contains the identification number, and not the personal data.

\section{The AGALZ agents in operation}

The objectives of AGALZ agents are: to plan the nurses and doctors working time dynamically, to maintain the standard working reports about their activities, and to guarantee that the patients assigned to the nurses are provided with suitable care. Thus the AGALZ agent schedules the working days obtaining dynamic plans depending on the tasks needed for each assigned patient. As can be seen in Fig. 3, the AGALZ nurse agent has five capabilities and offers three services.

AGALZ implements the reasoning cycle of the CBP system by means of three capabilities: Update, KBase and VCBP (Variational CBP). The Update capability implements the retrieve and retain stages, while the KBase capability implements the reuse stage and the VCBP capability the revise stage, where the nurse opinion is evaluated. The VCBP capability is also in charge of dynamic replanning task. By means of its Give Care capacity, AGALZ supervises each care task and generates the corresponding report. The Consult Nurse Data capability allows AGALZ to execute different queries on stored data.

Given a set of beliefs $B$ compatible with the problem $E$, it is possible to generate a plan base CBP that contains all the possible plans produced by the combinations of compatible beliefs. The beliefs available for the AGALZ agent are tasks, resources and time. 


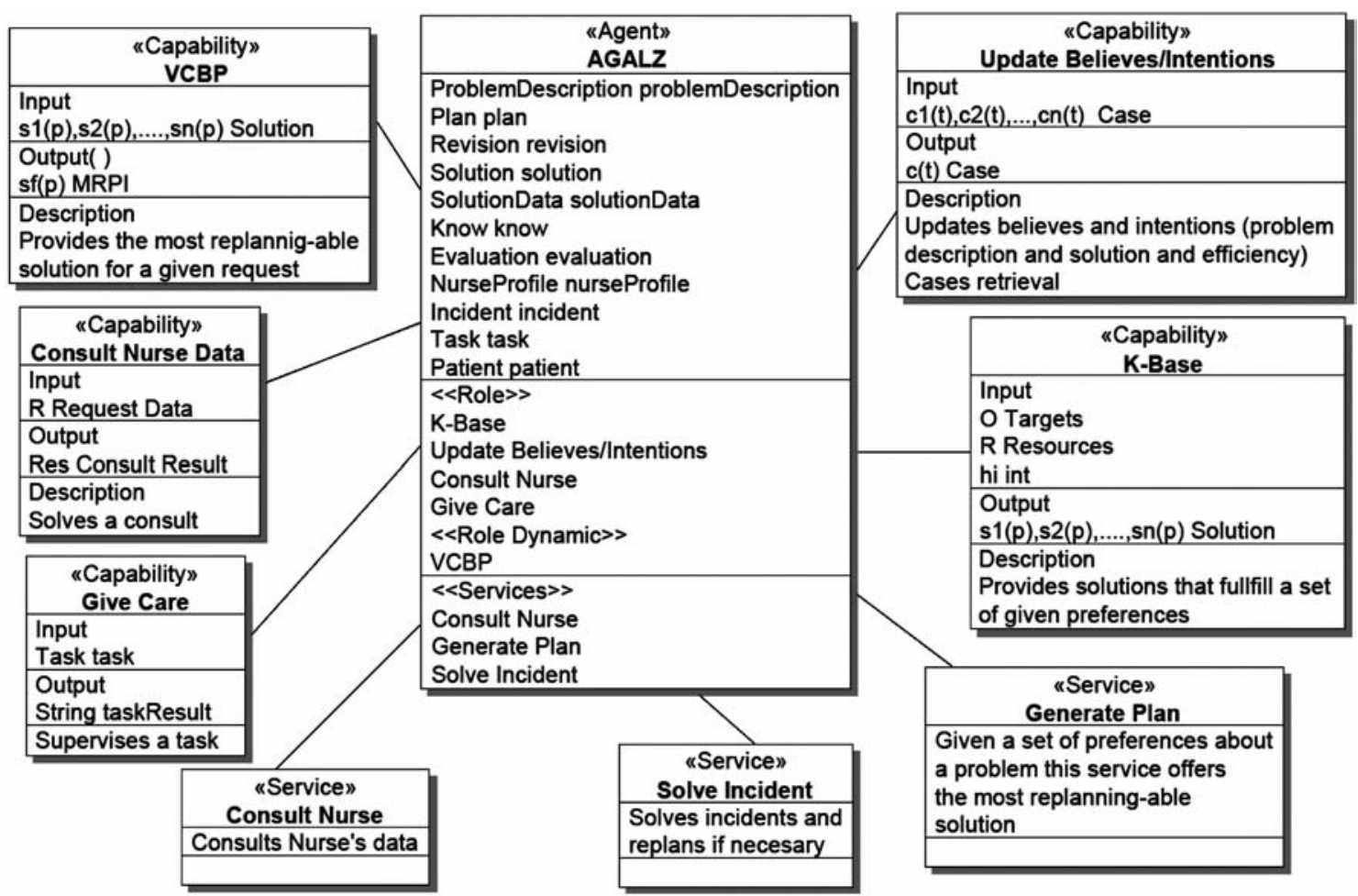

Fig. 3. AGALZ AUML class diagram.

675 A task is a java object that contains the data of the 676 patient who requested the service, the description of the 677 service and the time limits to carry it out, as can be seen 678 in Table 1. For each task one or more goals are 679 established, in such a way that the whole task is 680 eventually achieved. A goal is also a java object, that 681 identifies what the AGALZ agent wants to achieve 682 (complete a task) and under which conditions (restric683 tions). For this, a goal can contain parameters and define 684 creation conditions (that allow AGALZ to define the 685 conditions for achieving the goal), context conditions 686 (the conditions that must be fulfilled) or drop conditions. 687 To achieve its objectives each goal triggers plans. A plan 688 is a procedure written in java code. A goal can create 689 new goals (subgoals) to achieve its objectives (for 690 example for the task of rehabilitation AGALZ creates a 691 new goal for each concrete exercise).

692 The CBP system constructs plans as a sequence of 693 tasks that need to be carried out by a nurse. A 694 description of the problem will be formed by the tasks 695 that the nurse needs to execute, the resources available, 696 and the times assigned for their shift. In the Update 697 stage, the descriptions of similar problems are recov698 ered. In order to do this, the AGALZ agent allows the 699 application of various similar algorithms (cosine, 700 clustering etc.). In this step, those problem descriptions found within a range of similarity close to the original problem description are recovered from the beliefs base. In our case, a tolerance of $20 \%$ has been permitted.

Once the most similar problem descriptions have been recovered, the K-Base capability recovers the solutions associated with them. One solution contains all the plans (sequences of tasks) that are carried out in order to achieve the objectives of AGALZ for a problem description (assuming that replanning is possible) in the past, as well as the efficiency of the solution being supplied.

Table 1

Task example

\begin{tabular}{ll}
\hline Task & Data \\
\hline TaskId & 36 \\
TaskType & 32 \\
TaskDescript & Feeding (lunch) \\
TaskPriority & 3 \\
TaskObjective & 0 \\
TaskIncidents & 0 \\
PatientId & 7 \\
PatientDependence & 2 \\
MinTime & $12: 30$ \\
MaxTime & $15: 00$ \\
TaskResources & Food \\
\hline
\end{tabular}

$\mathrm{t} 1.3$

t1. 4

t1.5

$\mathrm{t} 1.6$

t1.7

t1.8

t1.9

t1.10

t1.11

t1.12

t1.13

t1.14 
The VCBP capability also combines the recovered solutions, as explained in Section 4, to construct a plan. At this time AGALZ takes control of the processing of the plan (scheduling). The VCBP capability is centered around the objectives and resources needed by each task, as well as on the objectives that the nurse needs to perform and the resources available in order to carry out the global plan. The objectives or global plans that each nurse has are to attend to the patients and not to work for over $8 \mathrm{~h}$. The time available is a problem restriction. This available time will influence the hyper plan of restrictions, specifically, the range of positive values that the $z$ axis takes from this hyper plan. The resources necessary for some of the tasks are food, equipment and rooms. Finally, the VCBP capability takes care of incidents and interruptions that may occur during replanning.

In order to illustrate how the planner works, let's take a significant example. In the first place it is necessary to take into account that each nurse has a different profile according to their qualification and the tasks that they usually carry out. Let $\mathrm{pr}=\mathrm{pr}_{1}, \cdots, \mathrm{pr}_{10}$ define the stored profiles of the nurses at the residence. It is considered appropriate to manage the profiles of the nurses because there are some nurses who perform tasks with greater skill or who carry out tasks in less time. On the other hand, the AGALZ agent maintains a close relationship with the Manager agent. The Manager agent has as one of its tasks the assignation of nurses to patients and doctors to patients. This assignation is carried out through the CBR reasoning motor of the Manager Agent. When the new assignation of tasks needs to be carried out to the nurses or to the doctors, both past experiences, such as the profile of the nurse or doctor, and the needs of the current situation are retrieved. In this way tasks are allocated to a nurse. These tasks may correspond to the same patient or to a number of patients. Moreover, as mentioned above, the profile of each nurse is taken into account. For example, not all nurses are equally qualified for rehabilitation. If one nurse is more qualified in the area, she will be allocated the patients whose need for rehabilitation is greater, always taking into account that the nurse cannot work more than $8 \mathrm{~h}$, so that the number of patients assigned depends on the time needed to carry out the rehabilitation. The Manager agent takes into account how those patients who receive rehabilitation are improving, the arrival of new patients, holiday rotas etc. As such, the allocation of tasks needs to be set on a daily basis.

Secondly, it is necessary to store within the beliefs base the time that each task takes, described as $t_{j}=\operatorname{Max}_{j, k}\left\{t_{j k}^{i}\right\}$, where $j$ indicates the type of task, $k$, the nurse with the most suitable profile to carry it out (since it is only possible to assign on each task type to the nurses who are qualified to carry it out) and $i$, the patient that requires the task.

Once the assignation of tasks to a nurse has been completed, the assignation is communicated to the corresponding AGALZ agent. From this moment on, the planning process begins. The AGALZ agent must take into account the time that nurse has available and the time required for each task. Moreover, the resources available and the location of the patients involved are also taken into account. In order to make a plan, the cases with a similar problem are recovered from the beliefs base and solutions (plans) that were used to resolve them are combined.

A large quantity of measurements have be taken in order to standardise the time taken to arrive at a given room, or to take a patient from one room to another (depending on the level of dependence of the patient). These times are included directly in the time assigned for each task.

The location of the patients is a factor which significantly influences the decision as to whether a plan should be interrupted. For example, in the case that a nurse should go to a given room to take dinner to the patient and the patient is actually in a different room, the nurses plan will need to be interrupted. As mentioned above, the location of the patients within the hospital is defined through a reference system in $\mathfrak{R}^{2}$. In the location system, it is fundamental that RFID devices are used. These devices make it possible to rapidly assess the possibility or need to replan.

A plan can be interrupted for different reasons. Those which have been taken into account within the residence are: that a resource fails, that a patient suffers some sort of crisis and requires unforeseen attention, that the patient has an unexpected visit or that visits to the patient have gone on over the permitted time allowed and an emergency situation. If the planner finds itself in a situation where the plan is interrupted, it rejects the initial plan and seeks an alternative one. The first thing that needs to change is the task order, attempting to maintain the assignation originally allocated by the Manager agent. The new plan must meet the initial objectives. In the event that this is impossible, the nurses will need to be reassigned. This reassignment will attempt to limit changes to a minimum. For reassignment it is necessary to take into account the tasks that were assigned to the nurses, the development of the plans (which tasks have been carried out and which still need to be done) and the profiles of the nurses (prioritising preparation for the task that cannot be covered). The nurse who is assigned the task should replan in order to include the new task. In the event that 
816 the replanning is positive (the tasks that still need to be 817 done and the new task can be carried out) the process is 818 complete. If the replanning is negative, the next nurse 819 down in the ranking will be used.

820 Lastly, depending on the efficiency of the plan, it will 821 be stored together with its level of efficiency within the 822 beliefs base. In the paragraphs below, we give a specific example in detail.

Let $E^{i}=\left\{e_{0}^{i}, \cdots, e_{h}^{i}\right\}$ the task carried out on patient $i$, put in order of priority. We have the following problem $E=\cup_{i} E^{i}=\left\{e_{0}, \cdots, e_{n}\right\}$, that is updated on a daily basis, where $E$ denotes the complete set of tasks being carried out and therefore has no superscript.

Selecting a nurse $k \in\{1, \cdots, 10\}$ at random (in particular, $k=3$ ), it has been shown that the assignation of tasks according to the profile was:

- Take patient 2 to the toilet $\equiv e_{1}^{2} ; t_{1}=30 \mathrm{~min}$.

- Wash patient $2 \equiv e_{3}^{2} ; t_{1}=30 \mathrm{~min}$.

- Give patient 2 their breakfast $\equiv e_{2}^{2}, t_{2}=20 \mathrm{~min}$.

- Give patents 4, 5 and 6 rehabilitation $e_{4}^{5}, e_{5}^{5}, y e_{5}^{6}$; $t_{5}=90 \mathrm{~min}$.

- Check weight, blood pressure etc. of patient $6 \equiv e_{0}^{6}$; $t_{0}=60 \mathrm{~min}$.

- Wash patient $5 \equiv_{3}^{5} ; t_{3}=30 \mathrm{~min}$.

- Check weight, blood pressure etc. of patient $5 \equiv e_{0}^{5}$; $t_{0}=60 \mathrm{~min}$.

Calculation of the tasks assigned verifies that the total time allocated does not go over $8 \mathrm{~h}$. As may be noted, when the tasks are being assigned, the location of the patients is not taken into account (the patients are given the best treatment possible). But the location of the patients is taken into account when the plan is generated (in order to minimise the total time taken to carry out the tasks).

Once the assignation of tasks is complete, each ALGALZ agent carries out a plan for its nurse. They retrieve similar assignations from the beliefs base, and the corresponding plans that were used. A plan is made and supplied to the nurse. The nurse then carries out the plan in sections, in other words, task by task (The current task is shown in the PDA and the nurse has to introduce the result obtained after the task has been accomplished). Each task has a series of objectives which must be reached for the part of the plan to have been completed successfully. In order to carry out each task the nurse must have a number of resources available. For example, the task "Check weight, blood pressure etc." corresponds to the objective "checking health of patient" $\equiv O_{0}$; the tasks "Take patient to the toilet" and "wash patient" corresponds to the objective of appearance and physical well-being $\equiv O_{1,3}$ and breakfast, lunch, tea and dinner correspond to the objective, physical recuperation $\equiv O_{2,4,6,7}$ (task 2 indicates breakfast, task 4 indicates lunch, task 6 indicates tea, and 7 indicates dinner). The coding used for resources is similar. It has been decided that the objectives and resources variables should be dichotomic (binary) with a value of 0 to 1 in order to indicate the absence or presence of a resource or objective and to be represented in Fig. 4a. Value 1 indicates that this resource is needed or that this is the objective to be reached, while zero denotes the contrary.

Fig. 4a shows the representation of a space $\mathfrak{R}^{3}$ for tasks according to the following three coordinates: time, number of objectives achieved, and number of resources used (coordinates taken from similar cases recalled). Specifically, Fig. 4a shows a hyper plan of restrictions and the plan followed for a case retrieved from the beliefs base, considered to be similar. So that Fig. $4 \mathrm{a}$ isn't overly large, and in order for the plan to be appreciated at first glance, the time axis has been
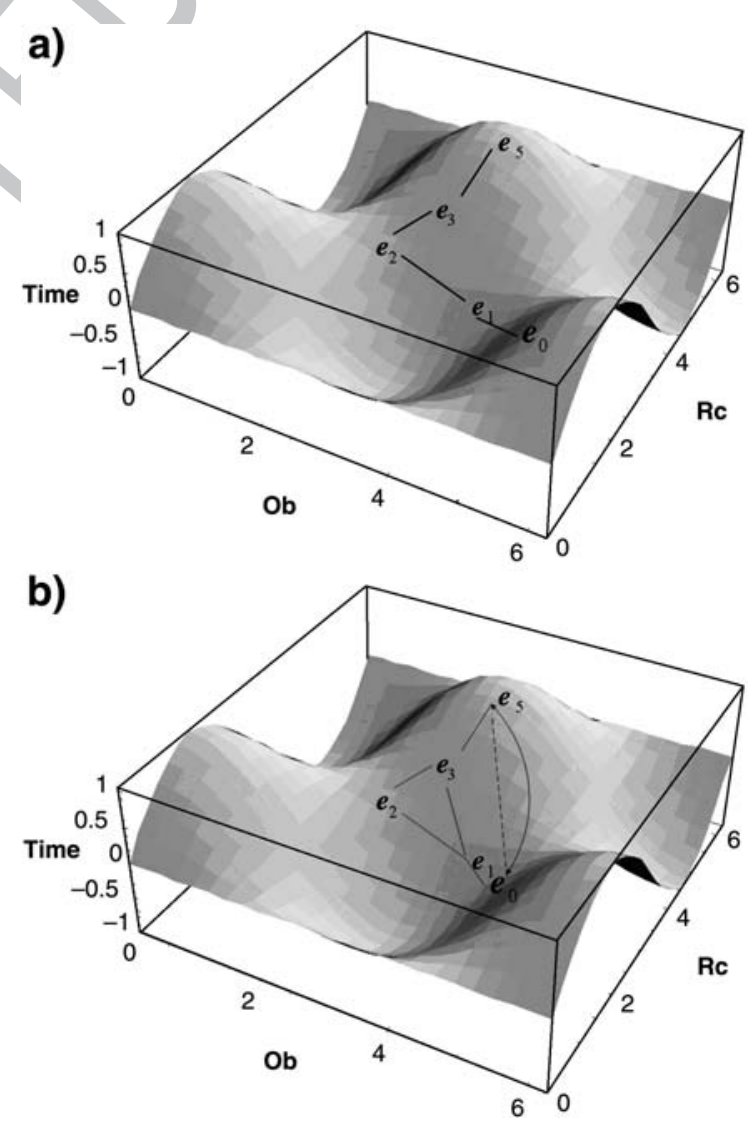

Fig. 4. Hyperplan of restrictions. a) Hyperplan together with the corresponding plan. b) Selection of the most replannable plan. 
888 rescaled (axis $z$ ), establishing an isomorphism between 889 the intervals [0.1] and [0.8]:

890 The isomorphism is as follows:

891

$\lambda:[0,1] \rightarrow[0,8]$
$z \rightarrow \lambda(z)=8 z$

893 For other similar retrieved cases, the same procedure is 894 followed. The new plan is made in such a way that the 895 planner proposes the plan in sections, with the greatest 896 density of plans around it (reflected by formulae (15) and 897 (16)). In short, from the tasks that the nurse needs to carry 898 out for one or several patients, the most similar cases 899 (from past experiences) are retrieved from the beliefs 900 base. Below, the hyper plan and the plan carried out are 901 shown. In order to understand the graphical representa902 tion, given that the plans are made in sections, we focus on 903 one initial task $e_{0}$ and a final task $e_{5}$ on the same or a 904 different patient. Between the initial and the final task the 905 nurse could carry out other tasks (that involves the same 906 patients as those corresponding to tasks $e_{0}$ and $e_{5}$ or that 907 implicate other patients). The idea that the planner 908 presents is to choose as the optimal solution the plan 909 that has the most plans around it, involving these two 910 fixed tasks on the patient/s assigned, (independently of 911 whether or not it includes other tasks for other patients, if 912 the task is for a patient that hasn't been assigned to the 913 nurse, they are not carried out, but in this way, it also 914 allows the intersection of tasks on other patients):

915 In this way, as can be seen in Fig. 4b, the plan chosen is 916 the one represented by a discontinuous line since it 917 represents the plan that has most other plans around it and 918 involves other tasks that could be assigned in the case of 919 interruptions. In the example, the plan proposed was:

- Take patient 2 to the toilet $\equiv e_{1}^{2} ; t_{1}=30 \mathrm{~min}$.

- Wash patient $2 \equiv e_{3}^{2} ; t_{3}=30 \mathrm{~min}$.

- Give patient 2 breakfast $\equiv e_{2}^{2}, t_{2}=20 \mathrm{~min}$.

- Give rehabilitation to patients 4 and $5 \equiv e_{5}^{4} y e_{5}^{5}$; $t_{5}=90 \mathrm{~min}$.

- Check patient 5's weight, blood pressure etc. $\equiv e_{0}^{5}$; $t_{0}=60 \mathrm{~min}$.

- Wash patient $5 \equiv e_{3}^{5} ; t_{3}=30 \mathrm{~min}$.

- Give rehabilitation to patient $6 \equiv e_{5}^{6} ; t_{5}=90 \mathrm{~min}$

- Check patient 6 's weight, blood pressure etc. $\equiv e_{0}^{6}$; $t_{0}=60 \mathrm{~min}$.

During the experiment, patient 5 suffered a crisis and needed to be attended to by the doctor. The nurse chosen was giving rehabilitation to patient 4 at the time. Once they had finished, patient 5 was still being attended by the doctor (ascertained by the location of the patient and the doctor), the time taken to attend to such a crisis is stored in the beliefs base.

The replanning applied took into account the tasks that still needed to be done and the time considered necessary for attending the crisis. In this way the planner proceeded to reorganise and replan the plan as follows:

- Give rehabilitation to $4 \equiv e_{5}^{4} ; t_{5}=90 \mathrm{~min}$.

- Give rehabilitation to $6 \equiv e_{5}^{6} ; t_{5}=90 \mathrm{~min}$.

- Check patient 6's weight, blood pressure etc. $\equiv e_{0}^{6}$; $t_{0}=60 \mathrm{~min}$.

- Check patient 5's weight, blood pressure etc. $\equiv e_{0}^{5}$; $t_{0}=60 \mathrm{~min}$.

- Give rehabilitation to $5 \equiv e_{5}^{5} ; t_{5}=90 \mathrm{~min}$.

- Wash patient $5 \equiv e_{3}^{5} ; t_{3}=30 \mathrm{~min}$.

In the example presented, it has been possible to replan. In the event that it had been impossible to reorganise the tasks that remained, communication would be made with the Manager agent. The Manager agent would need to reassign the tasks according to the level of expertise of the nurses.

The mathematical calculations for obtaining $h(x)$, through Duchon techniques, the set of geodesics $\left\{g_{n}(x)\right\}_{n \in N}$ through the resolution of the Euler and transversability equations, or for obtaining the Jacobi field, are carried out using the programme ${ }^{\mathbb{B}}$ Mathematica 5.1 and the libraries Jspline+ and Jlink for java.

\section{Results and conclusions}

The ALZ-MAS system, incorporating AGALZ agents, has been tested over the last few months. During the testing period the system usefulness has been evaluated from different points of view. Fig. 5 shows the average number of nurses working simultaneously (each of the $24 \mathrm{~h}$ of the day) at the Residence before and after the implantation of the system prototype, with data collected from October 2005 to March 2006. The prototype was adopted on January 15th, 2006. The average number of patients was the same before and after the implementation. To test the system 30 patient agents, 10 AGALZ nurse agents, 2 doctor agents and 1 manager agent were instantiated. In the tests related to the frame of this research we have focused on the AGALZ nurse agents, while the doctor agents do not have planning capabilities incorporated within them as yet. As can be seen in Fig. 5, the dark-blue area represents the average number of nurses required in the residence each hour of a day without the ALZ-MAS. The light-blue area represents the same measure but after the implementation of the ALZ-MAS. As can be 
1

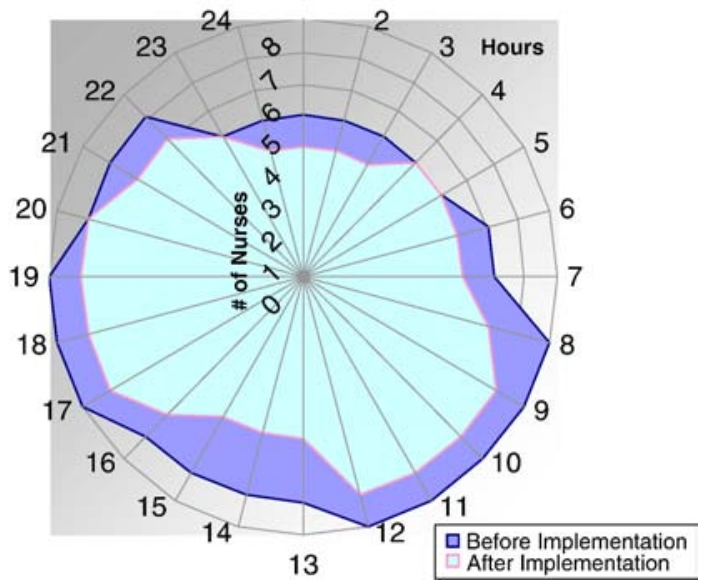

Fig. 5. Number of nurses working simultaneously. (For interpretation of the references to colour in this figure legend, the reader is referred to the web version of this article.)

986 seen, the ALZ-MAS helps the nurses to gain time, 987 which can be dedicated to the care of special patients, to 988 learn or to prepare new activities. The time spent on 989 supervision and control tasks has been reduced 990 substantially, as well as the time spent attending false 991 alarms, while the time for direct patient care has been 992 increased.

993 The tasks executed by nurses were divided in two 994 categories, direct action tasks and indirect action tasks. 995 Direct action tasks are those which require the nurse 996 acting directly on the patient during the whole task 997 (medication, posture change, toileting, feeding, etc.). In 998 the indirect action tasks the nurses do not need to act 999 directly on the patients all the time (reports, monitoring, 1000 visits). AGALZ agents can take care of some of these 1001 indirect actions, so nurses can dedicate more time to 1002 personal patients care. During the first testing period the 1003 problem was analysed and data was collected. The 1004 average time spent by nurses carrying out their duties 1005 with a given patient was obtained, having into account 1006 the patient type, its dependency level and the nurse 1007 professional level. For the direct action tasks, the 1008 following times were obtained for each patient: 100935 min cleaning, 18 min feeding, 8 min oral medication, $101030 \mathrm{~min}$ parenteral medication, $25 \mathrm{~min}$ posture change, $10118 \mathrm{~min}$ toileting, $60 \mathrm{~min}$ exercise and $10 \mathrm{~min}$ others for 1012 patients with a dependence degree of 1; and $45 \mathrm{~min}$ 1013 cleaning, $28 \mathrm{~min}$ feeding, $11 \mathrm{~min}$ oral medication, $101442 \mathrm{~min}$ parenteral medication, $50 \mathrm{~min}$ posture change, $101530 \mathrm{~min}$ toileting, $90 \mathrm{~min}$ exercise and $10 \mathrm{~min}$ others for 1016 patients with a dependence degree of 2 . We are 1017 especially interested on time spent on indirect tasks; 1018 daily times obtained before and after the implementation
Table 2

Time (min) spent on indirect tasks

\begin{tabular}{llllll}
\hline & Monitoring & Reports & Visits & Other & Total \\
\hline Before & 167 & 48 & 73 & 82 & 370 \\
After & 105 & 40 & 45 & 60 & 250 \\
\hline
\end{tabular}

for each task can be seen on Table 2. Table 2 shows how the implementation of the ALZ-MAS reduces the time spent on indirect task. For example, the average number of minutes spent by a nurse on monitoring patients has been reduced from 167 daily minutes to 105 daily minutes without reducing the care level and the patients safety.

Some authors such as Langer [10] have studied the role of the mindfulness in treating elderly people. During this research project we have been trying to construct a patient-centered social system. Both AGALZ and ALZ-MAS have been designed and developed from the perspective of the patients and the relationship established between the patients and staff. One of the main contributions of this paper is a dynamic planning mechanism which allows replanning in execution time, which in turn improves patient care. The system also facilitates the more flexible assignation of the working shifts at the residence; since the workers have reduced the time spent on routine tasks and can assign this time to extra activities, such as exercising the patients, learning, carrying out leisure activities or just talking with the patients or with their families. Their work is automatically monitored, as well as the patients' activities. The stored information may be analysed with knowledge discovery techniques and may help to improve the quality of life for the patients and the efficiency of the center. The security of the center has also been improved in three ways: the system monitors the patients and guarantees that each one of them is in the right place; secondly, only authorised personnel can gain access to the residence protected areas, and thirdly, the information is stored in a more secure way using redundance and generating continuous backups. The access to information has been protected in order to guarantee confidentiality.

We had certain problems implementing the system, partly because the nurses and workers were not familiar

Efficiency and quality of the CBP engine

\begin{tabular}{llllll}
\hline Strategy & \multicolumn{2}{l}{ Typical case } & \multicolumn{3}{c}{ Quality } \\
\hline $\mathrm{Ce}_{t}$ & $n_{\mathrm{c}}$ & $e_{\mathrm{fo}}$ & $e_{\mathrm{ffr}}$ & $e_{\mathrm{ffr}}$ & $\mathrm{Ce}_{0.2}$ \\
94 & 12 & 0.47 & 0.69 & 0.05 & 30 \\
\hline
\end{tabular}


1057 with the use of PDA devices, so some courses were given 1058 to introduce them to these technologies and teach them 1059 how to use the system interface. After that and with some 1060 difficulties with the installation of the wireless access 1061 points (with the propagation of the signal) and the 1062 collocation of the RFID door readers, the system was 1063 running smoothly, with only minor problems.

1064 The CBP-BDI architecture of the AGALZ gents 1065 presented in this paper solves one of the problems of 1066 BDI (deliberative) architectures, which is the lack of 1067 learning capacity. Table 3 shows achieved objectives vs. 1068 Possible objectives (efficacy: $e_{\mathrm{fo}}$ ); objectives reached 1069 vs. Resources used (efficiency: $e_{\mathrm{ff}}$ ); number of actions 1070 or beliefs used (plan stages: $n_{\mathrm{c}}$ ); efficiency of the plan in 1071 terms of the number of stages $\left(e_{\mathrm{ffr}}\right)$; percentage of cases 1072 that reach a solution state vs. the total number $\left(\mathrm{Ce}_{t}\right)$ and 1073 percentage of successful cases that reach values within 1074 the top $20 \%\left(\mathrm{Ce}_{0.2}\right)$. The agent improves its learning as 1075 the CBP system comes into play. The number of 1076 interruptions for replanning is notable reduced. It also 1077 reduces the gap that exists between the formalization 1078 and the implementation of BDI agents. The reasoning 1079 cycle of the CBR systems helps the agents to solve 1080 problems, to adapt to changes in the environment, and to 1081 identify new possible solutions. In order to evaluate the 1082 learning capacity of the AGALZ agents, the quality of 1083 the plans has been measured. The number of interrup1084 tions indicates the number of replannings carried out up 1085 to the completion of a plan. As previously explained in 1086 Section 2, AGALZ executes CBP cycles in order to 1087 learn. After the AGALZ agent executes 100 plans it 1088 reduces the number of interruptions, at an average of $108930 \%$. The average number of interruptions is of 9 times, 1090 per day, after ten executed plans, around 8 times after 1091 executing 50 plans and around 7 times after executing 1092100 plans. The results obtained lead us to conclude that 1093 AGALZ improves its behaviour with learning, and that 1094 the number of interruptions does not decrease by more 1095 than 7 per day, on average.

1096 In the future, health care for Alzheimer's patients, the 1097 elderly and people with other disabilities will require the 1098 use of new technologies that allow medical personnel to 1099 carry out their tasks more efficiently. Weick [17] 1100 describes the fundamental problems of knowledge 1101 transfer and sense making in digital/computer based 1102 environments. We have shown the potential of deliber1103 ative AGALZ agents in a distributed multi-agent system 1104 focused on health care, providing a way to respond to 1105 some challenges of health care, related for example to 1106 the identification, control and health care planning. In 1107 addition, the use of RFID technology on people 1108 provides a high level of interaction among users and patients through the system and is fundamental in the construction of the intelligent environment. Furthermore, the use of mobile devices, when used well, can facilitate social interactions and knowledge transfer.

\section{Acknowledgements}

This work has been partially supported by the MCYT TIC2003-07369-C02-02 and the JCYL-200205 project SA104A05. Special thanks to Sokymat by the RFID technology provided and to Telefónica Móviles (Movistar) for the wireless devices donated.

\section{References}

[1] A. Aamodt, E. Plaza, Case-based reasoning: foundational issues, methodological variations, and system approaches, AI Communications 7 (1994) 39-59.

[2] R.E. Bellman, Dynamic Programming, Princeton University Press, Princeton, NJ, 1957.

[3] M.E. Bratman, Intentions, Plans and Practical Reason, Harvard University Press, Cambridge, MA, 1987.

[4] L. Camarinha-Matos, H. Afsarmanesh, Design of a virtual community infrastructure for elderly care, in: L.M. CamarinhaMatos (Ed.), Proceedings of PRO-VE'02, Sesimbra, Portugal, 2002

[5] J.M. Corchado, R. Laza, Constructing deliberative agents with case-based reasoning technology, International Journal of Intelligent Systems 18 (2003) 1227-1241.

[6] K. Decker, J. Li, Coordinated hospital patient scheduling, in: Y. Demazeau (Ed.), Proceedings of ICMAS98, Paris, France, 1998, pp. 104-111

[7] D. Foster, C. McGregor, S. El-Masri., A survey of agent-based intelligent decision support systems to support clinical management and research, in: G. Armano, E. Merelli, J. Denzinger, A. Martin, S. Miles, H. Tianfield, R. Unland (Eds.), Proceedings of MAS*BIOMED'05, Utretch, Netherlands, 2005.

[8] M. Glez-Bedia, J.M. Corchado, A planning strategy based on variational calculus for deliberative agents, Computing and Information Systems Journal 10 (1) (2002) 2-14.

[9] L.T. Kohn, J.M. Corrigan, Donaldson, To Err is Human: Building a Safer Health System, Committee on Quality of Health Care in America Institute of Medicine, National Academy Press, Washington, DC, 1999.

[10] E.J. Langer, Mindfulness, Perseus Books, 1989.

[11] G. Lanzola, L. Gatti, S. Falasconi, M. Stefanelli, A framework for building cooperative software agents in medical applications, Artificial Intelligence in Medicine 16 (3) (1999) 223-249.

[12] T.R. McLean, Crossing the quality of chasm: a new health system for the 1st century, Health Matrix 12 (2002) 239-295.

[13] J.A. Meunier, A virtual machine for a functional mobile agent architecture supporting distributed medical information, in: I.E. E.E Computer Society (Ed.), Proceedings of CBMS '99, Washington, DC, USA, 1999.

[14] S. Miksch, K. Cheng, B. Hayes-Roth, An intelligent assistant for patient health care, Proceedings of Agents'97, ACM Press, New York, 1997, pp. 458-465).

[15] J. Nealon, A. Moreno, Applications of Software Agent Technology in the Health Care domain, Birkhauser, Whitestein series in Software Agent Technologies, 2003.
1119 
1164 [16] Sokymat, http://www.sokymat.com2006.

1165 [17] K.E. Weick, Cosmos vs. chaos: sense and nonsense in electronic 1166 contexts, in: R.L. Ruggles (Ed.), Knowledge Management Tools, 1167 vol. 3, 1985.

1168 [18] M. Wooldridge, N.R. Jennings, D. Kinny, The gaia methodology 1169 for agent-oriented analysis and design, Journal of Autonomous $1170 \quad$ Agents and Multi-Agent Systems 3 (3) (2000) 285-312.

Juan M. Corchado (PhD.). Received a PhD. in Computer Science from the University of Salamanca in 1998 and a PhD. in Artificial Intelligence (AI) from the University of Paisley, Glasgow (UK) in 2000. At present he is an Associate Professor, Director of the Intelligent Information System Group (http://bisite.usal.es) and Director of the MSc programs in Computer Science at the University of Salamanca (Spain), previously he was a sub-director of the Computer Science School at the University of Vigo (Spain, 1999-00) and Researcher at the University of Paisley (UK, 1995-98). He has been a research collaborator with the Plymouth Marine Laboratory (UK) since 1993. He has led several Artificial Intelligence research projects sponsored by the Spanish and European public and private institutions and has supervised seven $\mathrm{PhD}$. students. He is the co-author of over 130 books, book chapters, journal papers, technical reports, etc. published by organisations such as Elsevier, IEEE, IEE, ACM, AAAI, Springer Verlag, Morgan Kaufmann, etc., most of these present practical and theoretical achievements of hybrid AI and distributed systems. He has been the President of the organising and scientific committee of several international symposiums.
Javier Bajo (PhD. Student). At present he is an Assistant Professor at the University of Salamanca (Spain). He obtained an Information Technology degree at the University of Valladolid (Spain) in 2001 and an Engineering in Computer Sciences degree at the Pontifical University of Salamanca in 2003. He has been a member of the organising and scientific committee of several international symposiums such as CAEPIA, IDEAL, HAIS, etc. and co-author of papers published in recognized journal, workshops and symposiums.

Dante Tapia ( $\mathrm{PhD}$. Student). At present he is a $\mathrm{PhD}$. student at the University of Salamanca (Spain) under the supervision of Dr. Juan M. Corchado. He obtained an Engineering in Computer Sciences degree in 2001 and an MSc in Telematics at the University of Colima (Mexico) in 2004. He has been involved in the development of automated systems in the Faculty of Telematics at the University of Colima and deeply collaborating with the Government of the State, where he obtained a scholarship to complete his academic formation. He has also been a coauthor of papers published in recognized workshops and symposiums.

Yanira de Paz (PhD. Student). At present she holds a scholarship provided by the Spanish Minister of Education to complete a $\mathrm{PhD}$. program at the University of Salamanca (Spain). She obtained a Mathematics degree in 2002 and a Statistic degree in 2003 at the University of Salamanca (Spain). She is an Assistant Professor at the Faculty of Economy at the University of Salamanca and co-author of several mathematical and statistical books. She has also been a lecturer in the Faculty of Mathematics at the Complutense University of Madrid. 